\section{¿NO LO}

\section{ENCUENTRA?}

\section{Búsquelo en el Directorio} Médico Español

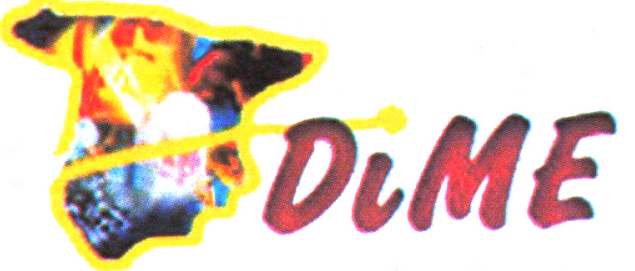

La más completa y actualizada base de datos que alberga información

referente a todos los recursos

nacionales, accesibles a través de Internet, que están relacionados con la Medicina y la Sanidad.

El DiME presenta la información sencillamente estructurada, aunque también ofrece la posibilidad de usar su potente motor de búsqueda, herramienta de inestimable ayuda para quienes prefieren realizar búsquedas más selectivas y dirigidas, ya sea por medio de una simple palabra clave o utilizando las intuitivas propiedades avanzadas de que dispone.

\section{Toda la información en}

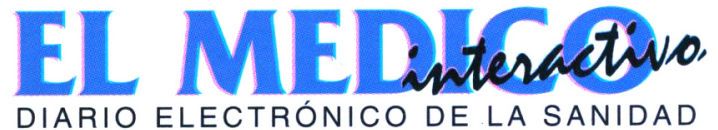

http://www.medynet.com/elmedico
1. DENOMINACIÓN DEL MEDICAMENTO Vastat Flas" $15 \mathrm{mg}$ y Vastat Flas $30 \mathrm{mg}$ COMPOSICIÓN CUALITATIVA Y CUANTITATIVA Cada comprimido contiene $15 \mathrm{mg}, 030 \mathrm{mg}$ de mirtazapina. Lista de excipientes en 6.1. 3. FORMA FARMACÉUTICA Comprimidos bucodispersables Los comprimidos de Vastat Flas son redondos, blancos y con bordes biselados, marcados con un código en una de las caras (TZ11, comprimidos $15 \mathrm{mg}$ y TZ/2, comprimidos $30 \mathrm{mg}$ ). 4. DATOS CLINCOS 4.1. Indicaciones terapéuticas Episodio de depresion mayor 4 . Posologia y forma de administración Para evitar que el comprimido se aplaste,

presione el alveolo Cada blister contiene 6 alvéolos separados por lineas pertoradas Doble el blister y separe un alveolo siguiendo las lineas pertoradas. Separe cuidadosamente la lámina del alveolo por la esquina indicada con una flecha. Saque el comprimido de su alveolo con las manos secas y póngaselo en la lengua. El comprimido se disgregara rápidamente y puede tragarse sin agua. Adultos: La dosis eficaz se encuentra normalmente entre 15 y $45 \mathrm{mg}$ al dia; el tratamiento se inicia con $15030 \mathrm{mg}$ (la dosis mas alla se tomara por la noche). Ancianos: La dosis recomendada es la misma que para los adultos. En pacientes ancianos el aumento de dosis debe realizarse bajo estrecha supervision para consegur una respuesta satsftactona y segura. Niños: No se ha determinado la eficacia y seguridad de Vastat en niños, por 10 tanto no se recomienda tratar nifíos con Vastat. El aclaramiento de mirtazapina puede disminuir en pacientes con insuticiencia hepatica 0 renal. Esto debe tenerse en cuenta cuando se prescribe Vastat a estos pacientes. Mirtazapina tiene una semivida de 20-40 horas, por lo que Vastat puede administrarse una vez al dia, preferiblemente como dosis única por la noche antes de acostarse. Tambien puede administrarse en subdosis divididas a partes iguales durante el dia (una por la mañana y una por la noche). Es recomendable continuar el tratamiento hasta que el paciente ya no presente sintomas durante 4-6 meses. Postenormente, el tratamiento puede abandonarse gradualmente. El tratamiento con una dosis adecuada deberá proporcionar una respuesta positiva en 2-4 semanas. Si la respuesta es insuficiente, la dosis puede aumentarse hasta la dosis máxima, pero si no se produce respuesta en otras 2.4 semanas, debe suspenderse el tratamiento. 4.3. Contraindicaciones Hipersensibilidad a la mirtazapina 0 a cualquiera de los excipientes. 4.4. Advertencias y precauciones especiales de empleo Durante el tratamiento con muchos antidepresivos, se ha descrito depresión de la médula ósea, que normalmente se presenta como

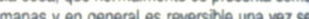
suspende el tratamiento. También se ha intormado de agranulocitosis reversible como acontecimiento adverso raro en estudios clinicos con Vastat. El médico deberá vigilar la aparición de sintomas como fiebre, dolor de garganta, estomattis u otros signos de infección; si se presentan tales sintomas hiebre, dolor de garganta, estomattis u otros signos de infeccion; si se presentan tales sintomas destablacuspenderse el tratamiento y realizarse un hernograma. En los siguientes casos es necesario y sindrome atectivo orgánico; a partir de la experiencia clinica parece que raramente se producen ataques en pacientes tratados con Vastat. Insuficiencia hepatica o renal. Entermedades cardiacas ataques en pacientes tratados con Vastat. Insuficiencia hepatica o renal. Entermedades cardiacas las que deberán tomarse las precauciones habituales y administrar con precaución los medicamentos concomitantes. Hipotensión. Al igual que con otros antidepresivos deben tomarse precauciones en pacientes que se encuentren en las siguientes situaciones: Aiteraciones de la micción como hipertrofia prostática (aunque en este caso no es de esperar que se produzcan problemas debido a que Vastat posee una actividad anticolinérgica muy débil). Glaucoma agudo de ángulo estrectho con presion intraocular elevada (en este caso también es muy poco probable que aparezcan problemas, porque Vastat tiene una actividad anticolinérgica muy débil). Diabetes melitus. El tratamiento debe suspenderse si se presenta ictencia. Además, al igual que con otros antidepresivos, deben tenerse en cuenta los siguientes factores: Puede darse un empeoramiento de los sintomas psicóticos cuando se administran antidepresivos a pacientes con esquizotrenia u otras alteraciones psicoticas; pueden intensificarse los pensamientos paranoides. Si se trata la fase depresiva de la psicosis maniacodepresiva puede revertir a la fase maniaca. Respecto a la posibilidad de suicidio en particular al inicio del tratamiento, debe proporcionarse al paciente, en casos particulares, un numero limitado de de remprimidos de Vastat Flas. Aunque los antdepresivos no producen adicción, la suspension brusca malestar Los pacientes ancianos son más trecuentemente sensibles, especialmenter a cabeza y malestar. Los pacientes ancianos son más hecuentemente sensibles, especiaimente a los etectos adversos do los antoepresios. Durante la investigacion cinica con vastat no se ha informado de la edad, sin embargo, la expenencia hasta el momento es limitada. Vastat Flas contiene aspartamo, que origina tenilalanina 105 comprimidos de 15 y $30 \mathrm{mg}$ contienen 2.6 y $5.2 \mathrm{mg}$ de tenilalanina, respectivamente. La fenilalanina puede ser dahina para los pacientes fenilcetonunicos. $\mathbf{4 . 5}$. Interacción con otros medicamentos y otras formas de interacción. Datos in vitro sugeren que Interaccion con otros medicamentos y otras tormas de interacción. Datos in vitro sugeren que la mirtazapina es un inhibidor competitvo muy debir de los enzimas CYPA2, CYP2O6 y CrP3A del Figura 1.

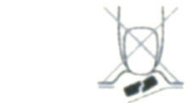
Figura 2a

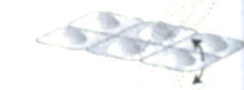

Figura 20 grado por la CYP1A2 En un estudio sobre interacciones en voluntarios sanos no se mostro influencia el efecto de un ine el electo de un inhibidor de la CYP3A4 en la farmacocinética de la mirtazapina in vivo. Deben supervisarse cuidadosamente tratamientos concomitantes con mirtazapina e inhibidores potentes de la CYP3A4, como inhibidores de la proteasa del HIV, antifúngicos azolicos, entromicina y nefazodona. La carbamazepina, que es un inductor de la CYP3A4 aumentó aproximadamente dos veces el aclaramiento de mirtazapina, to que resultó en una disminución de los niveles plasmáticos del 45-60\%. Si se añade la carbamazepina u otro inductor de metaboissmo de tarmacos (como nitampicina o lenitoina) a la lerapia con mirtazapina, puede ser necesanio aumentar la dosis de mirtazapina Si el tratamiento con el inductor se suspende, puede ser necesario disminuir la dosis de mirtazapina. La biodisponibilidad de la mirtazapina aumentó en más del $50 \%$ al administrarse conjuntamente con cimetidina. Puede ser necesario disminuir la dosis de mirtazapina en caso de iniciar un tratamiento concomitante con cimetidina o aumentarla cuando finaliza el tratamiento con este medicamento. En los estudios in vivo sobre interacciones, la mirtazapina no influyó en la farmacocinética de la risperidona ni de la paroxetina (sustrato de la CYP2D6) carbamazepina (sustrato de la CYP3A4), amitriptilina ni cimetidina. No se han observado efectos ni cambios clinicos relevantes en la tarmacocinética en humanos de la administración conjunta de mirtazapina y litio. Mirtazapina puede potenciar la acción depresiva del alcoho sobre el sistema nervioso central; por tanto los pacientes deben ser advertidos de que eviten el alcohol durante el tratamiento con Vastat Vastat no debe administrarse simultáneamente con inhibidores de la MAO ni en las dos semanas posteriores a la finalización del tratamientc con estos agentes. Mirtazapina puede potenciar los etectos sedantes de las benzodiacepinas; deben tomarse precauciones cuando $\mathrm{SE}$ prescriben estos tármacos junto con Vastat. 4.6. Embarazo y lactancia Aunque los estudios en animales no han mostrado ningun electe teratogénico con trascendencia toxicologica, no se ha establecido la seguridad de Vastat en el embarazo humano. Vastat se utilizara en $\theta$ embarazo unicamente si la necesidad es clara. Aunque los experimentos en animales muestran que mirtazapina se excreta en cantidades muy pequeñas por la leche, el uso de Vastat en mujeres que dan el pecho no es aconsejable porque no existen datos disponibles sobre th excrecion por la leche humana 4.7 . Efectos sobre la capacidad para conducir y utilizar máguinas Vastat puede disminuir la concentración y la alerta los pacientes en tramiento con antidepresivos deben evitas realizar actividades potenciaimente peligrosas que requieran ut estado de alor acientes en tratamiento con antidepresivos decentevitar reaizar activicades potenciaimente peligrosas que requieran depresion presentan varios sintomas recondicir un veniculo a motor manejar maquinaria. 4.8. Reacciones adversas Los pacientes con cepresion presentan vanios sintomas relacionados con la enflermedad misma. Por tanto, a veces es dificir diterenciar los sintomas que sol Vastat son: aumento de apetito y aumento de peso, somnolencia (que puede atectar negativamente a la concentración), generalmente duranti las primeras semanas de tratamiento (Nota: En general, la reducción de dosis no produce menor sedación sino que además puedi comprometer la eficacia antidepresiva), edema generalizado o local, con aumento de peso, mareo, cetalea. En casos raros $10,01-0,1 \%$

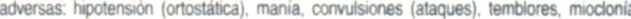
depresion aguda de la médula ósea (eosinoflia, granulocitopenia, agranulocitosis, anemia aplasica y trombocitopenia) (ver tambien seccior 4.4 "Advertencias y precauciones especiales de emple $0^{\text {") }}$, aumento en las actividades de las transaminasas séricas, exantema, parestesia sindrome de las piernas inquietas, artralgia / mialgia, tatiga, pesadillas / suentos intensos. 4.9. Sobredosis La experiencia hasta el momentr (aunque todavia limitada) respecto a sobredosificación con Vastat solo, indica que los sintomas son en general leves. Se ha descrito depresión del sistema nervioso central con desorientación y sedación prolongada, junto con taquicardia e hiper o hipotensión leves. Los casos de sobredosificación deberán tratarse mediante lavado gástrico, conjuntamente con una terapia sintomática apropiada y de apoyo de las funciones vitales. 6. DATOS FARMACÉUTICOS 6.1. Lista de excipientes Vastat Flas 15 y $30 \mathrm{mg}$ comprimidos contene: Azucar en esteras hidroxipropilmetilcelulosa, povidona, estearato de magnesio, copolimero $\mathrm{E}$ de amiloalquilmetacrilato (Eudragit E100), aspartamo (E 951), ácido citrico, crospovidona, manitol, celulosa microcristalina, aroma de naranja natural y artificial y bicarbonato sódico. 6.2 Incompatibilidades № aplicable 6.3. Periodo de validez 2 años.6.4. Precauciones especiales de conservación Vastat Flas debe conservarse en su envase original. 6.5. Naturaleza y contenido del recipiente Los comprimidos de Vastat Flas se envasan en blisters a prueba de niños, rigidos, del tipo que debe separarse la lámina para abrirlos. Están formados por un laminado de lámina de aluminio y peliculas plasticas, selladas a otro laminado de papel y lámina de aluminio recubierta con una laca resistente al calor. Los alvéolos de los comprimidos están separados por lineas pertoradas. Las peliculas plásticas contienen PVC (cloruro de polivinilo). poliamida y poliéster. Están disponibles las siguientes presentaciones: Cada blister contiene 6 comprimidos. Envases de 30 comprimidos bucodispersables $(5 \times 6)$ de 15 mg de mirtazapina (código TZ/1). Envases de 30 comprimidos bucodispersables $(5 \times 6)$ de 30 mg de mirtazapina (codigo TZ/2). 6.6. Instrucciones de uso y manipulación Véase Apartado 4.2. 6.7. Código Nacional y precio venta público Con receta médica. Incluido en VASTAT FLAS $30 \mathrm{mg} 30$ comprimidos bucodispersables: CN: 770842. PVP+iva: 46.03€ 7. TITULAR DE LA AUTORIZACIÓN DE COMERCIALIZACIÓN Laboratorios Pensa Avda. Mare de Déu de Montserrat, 215-08041 Barcelona 8. NÚMERO(S) DE REGISTRO Vastat AUTORIZACIÓN O DE LA RENOVACIÓN DE LA AUTORIZACIÓN Julio 2002 10. FECHA DE LA REVISIÓN DEL TEXTO JUnio 2002. Bibliografia: 1. Benkert O. Muller M. Szegedi A. An overview of the clinical efficacy of mirtazapine. Hum Psychopharmacol Clin Exp 2002:17(Suppl 1):S23-S26. 2. Anttila SAK, Leinonen E. A review of the pharmacological and clinical profile of mirtazapine. CNS Drugs Reviews 7[3|,249-264.2001.3. De Boer Th. The pharmacologic profle of mirtazapine. J Clin Psychiatry 1996;57(suppl 4):19-25. OLaboratorios Pen S. A. 2004 


\section{Para pacientes con depresión, ansiedad asociada y alteraciones del sueño, que necesiten una rápida solución.}
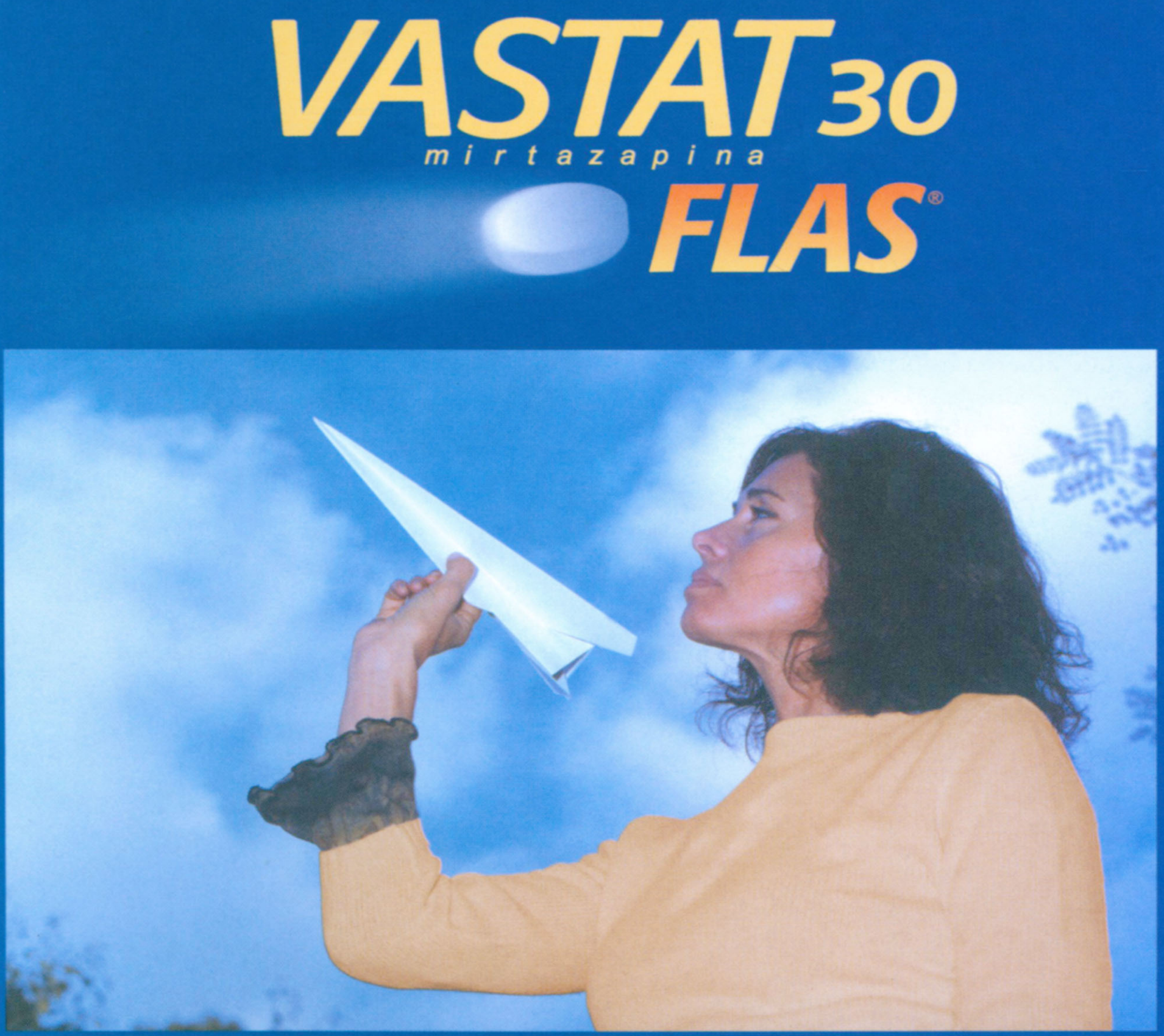

MEJORAR LA VIDA DIARIA DE SUS PACIENTES CON DEPRESIÓN ES

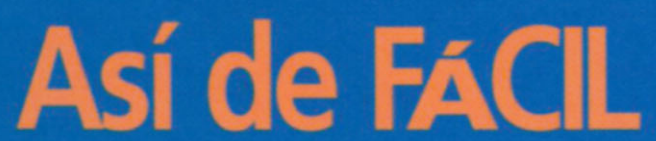

Antidepresivo de última generación 


\section{Para el tratamiento de la Depresión}

\section{y sus síntomas asociados, insomnio y ansiedad}
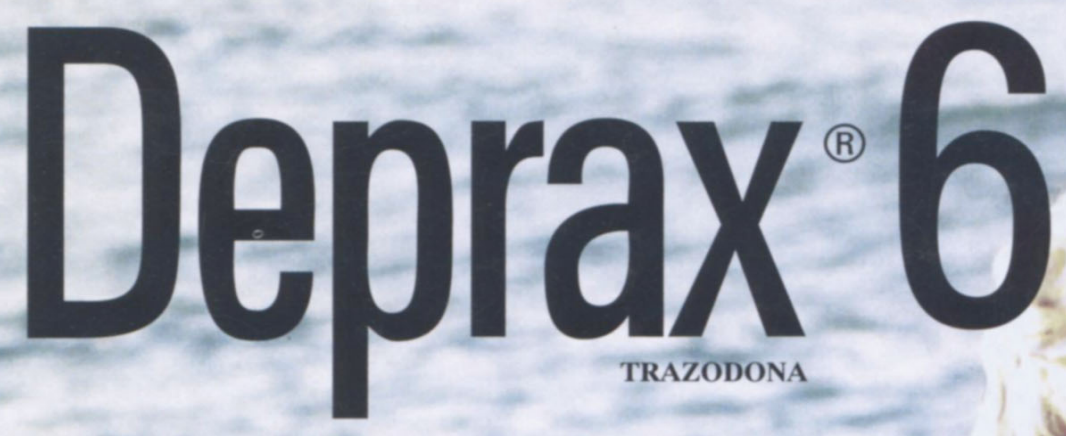

\section{Perfil farmacológico único}

El antidepresivo S.A.R.I., antagonista 5-HT

e inhibidor de la recaptación de serotonina

\section{Perfil clínico completo}

- Antidepresivo

- Hipnorregulador (1849)

- Ansiolítico
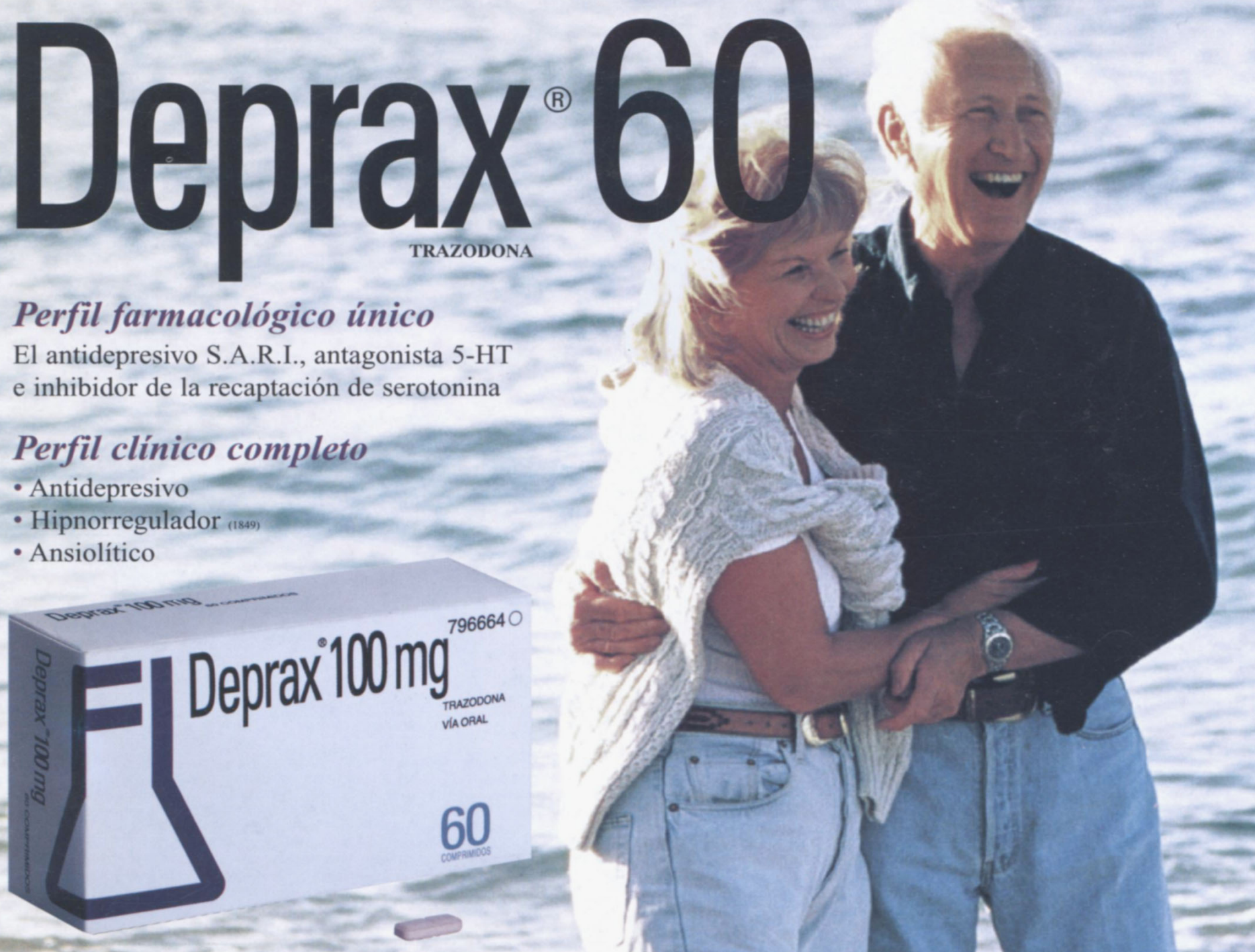

Deprax está constituido por trazodona clorhidrato, molécula con potente acción antidepresiva, que difiere de los habituales fármacos antidepresivos, tanto por su estructura química como por su mecanismo de acción. Los datos bioquímicos y neurofisiológicos localizan el lugar de actuación de Deprax a nivel de las estructuras diencefálicas que controlan y organizan la esfera emotivo-afectiva, cuya alteración primitiva o secundaria constituye un estadio patogénico común a la depresión y de sus equivalentes somáticos. COMPOSICIÓN. Cada comprimido contiene trazodona (DCI) HCI, 100 mg. Excipientes: cefulosa microcristalina, hidrógeno fosfato de calcio dỉhidrato, croscarmelosas sódica, povidona, rojo cochinilla A(E-124), amarillo anaranjado S (E-110), estearato magnésico, Eudragit E 12,5\%, talco micronizado, INDICACIONES. Psiquiátricas: depresiones orgánicas, endógenas, psicógenas, sintomáticas e involutivas. Estados mixtos de depresión con ansiedad. Depresiones atípicas o ernmascaradas (trastornos psicosomáticos). Neurológicas; temblores, đisquinesias, Geriátricas; trastornos emotivo-afectivos y de la conducta: irritabilidad, agresividad, labilidad emotiva, apatia, tendencia al aislamiento, đisminución en la duración del sueńo. Quirúrgicas: medicación preanestésica, preendoscépica y postoperatoria. POSOLOGíA. La dosificación se adaptará a al aislamiento, disminución en la duración del sueńo. Quiruirgicas: medicación preanestesica, preendoscópica y postoperatoria. POSOLOGIA. La dosificación se adaptará a las necesidađes y a la respuesta de cada paciente, empezándose por dosis bajas que se aumentarán progresivamente hasta los niveles adecuados y comenzando preferentemente. per la noche, En depresiones exógenas sintomáticas y psicogenas de mediana entidad: 150 a 300 mg/dia. En depresiones endógenas y psiccigenas severas: 300 a 600 mg/dia. c involutivas, indicaciones neurológicas y trastornos psicosomáticos; 75 a $150 \mathrm{mg} /$ dia. En geriatria: 50 a $75 \mathrm{mg}$ dia. NORMAS DE ADMIINISTRACIÓN. Se administrarín preferentemente después de las comidas. Caso de dosificación fraccionada desigual, la más elevada deberá administrarse por la noche. CONTRAINDICACIONES Y PRE. CAUCIONES. Se recomienda precaución en casos de insuficiencia cardiaca descompensada e hipotensión. Durante la primera semana de tratamicato puede influir en la capacidad para conducir vehiculos o mancjar maquinaria de precisión. No utilizar durante los tres primeros meses del embarazo. ADVERTENCLAS. Advertencias sobre excipientes: Este medicamento contiene rojo cochinilla A(E-124) y amarillo anaranjado S (E-110) como excipientes. Pueden causar reacciones de tipo alérgico, incluido asma, especialmente en pacientes lérgicos al ácido acetilsalicilico. INCOMPATIBILIDADES. No existen incompatibilidades. No obstante es aconsejable vigilar al paciente en caso de que se asocie a otros psicofármacos, así como en los tratamientos con antihipertensivos. EFECTOS SECUNDARIOS. En algunos casos puede aparecer somnolencia, astenia, hipotensión, cefalea, vértigo, ligera excitación, sequedad de boca, trastomos psicomotoresy gastrointestinales. En general, estos efectos son transitorios y no requieren la interrupeción del tratamiento. Como en otros fármacos dotados de acitividad alfa-adrenolitica, se han seńalado raros geasos de priapismo asociados al tratamiento con trazodona. En este caso, el paciente debe interrumpir el tratamiento.INTOXICACIÓN Y SU TRATAMIENTO. En caso de sobredosificación, debe instaurarse una lerapia sintomática mediante analépticos, lavado de estómago y diuresis forzada. PRESENTACTONY PVR Caja con 30 comprimidos de 100 mg: PVP IVA 4.-4,44 $€$. Caja con 60 comprimidos de $100 \mathrm{mg}$ : PVP IVA 4.- 8,12 €. Envase clínico: Caja con 1.000 comprimidos. OTRAS PRESENTACIONES, Cómprimidos: Caja con 30 comprimidos TA MÉDICA. INCLUIDO EN LA SEGURIDAD SOCIAL. APORTACIÓN REDUCIDA. BIBLIOGRAFiA: 177): Haria M., Fitton A., McTavish D., "Trazodone. A review of its pharmacology, therapeutic use in depression and therapeutic potential in othic físorders". Drugs \& Aging, 4 (4), 331335, 1994, 1849: Thase ME. "Antidepressant treatment of the depressed patient wi $335,1994$.

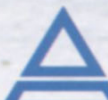

